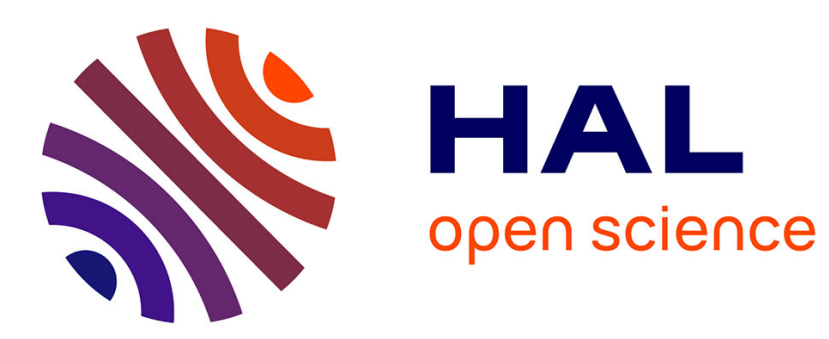

\title{
Exciton relaxation dynamics in quantum well heterostructures
}

\author{
M. Colocci, M. Gurioli, J. Martinez-Pastor
}

\section{To cite this version:}

M. Colocci, M. Gurioli, J. Martinez-Pastor. Exciton relaxation dynamics in quantum well heterostructures. Journal de Physique IV Proceedings, 1993, 03 (C5), pp.C5-3-C5-10. 10.1051/jp4:1993501 . jpa-00251499

\section{HAL Id: jpa-00251499 https://hal.science/jpa-00251499}

Submitted on 1 Jan 1993

HAL is a multi-disciplinary open access archive for the deposit and dissemination of scientific research documents, whether they are published or not. The documents may come from teaching and research institutions in France or abroad, or from public or private research centers.
L'archive ouverte pluridisciplinaire HAL, est destinée au dépôt et à la diffusion de documents scientifiques de niveau recherche, publiés ou non, émanant des établissements d'enseignement et de recherche français ou étrangers, des laboratoires publics ou privés. 


\title{
Exciton relaxation dynamics in quantum well heterostructures
}

\author{
M. COLOCCI, M. GURIOLI and J. MARTINEZ-PASTOR
}

\begin{abstract}
Dipartimento di Fisica e Laboratorio Europeo di Spettroscopie Non-Lineari, Largo Enrico Fermi 2, 50125 Firenze, Italia
\end{abstract}

\begin{abstract}
In this paper we report an overview of some points that we believe mostly relevant to the exciton dynamics in quantum well structures. We will concentrate our attention to the intrinsic radiative lifetime of the free excitons and the effects that localization has on the recombination kinetics. It is shown that a good agreement is eventually found between the theoretical predictions and the experimental data, even if some key aspects for a quantitative comprehension of the recombination mechanisms, namely the effects of phonon and/or interface defect scattering, still lack a complete understanding.
\end{abstract}

Exciton dynamics in semiconductor quantum wells $(\mathrm{QW})$ is being widely investigated; in fact it involves several important aspects and it can therefore provide many useful information on different physical processes, such as the energy-momentum and/or spin relaxation; exciton formation, ionization, trapping and localization; recombination kinetics; scattering by phonons, impurities or interface defects. Among this huge variety of dynamical effects, the exciton radiative recombination is probably the basic problem; nevertheless, only very recently one has begun to get a quantitative comprehension of the exciton recombination mechanism and still some aspects need further efforts to be fully elucidated.

The aims of this paper are to give a brief overview of some topics which we believe have been so far understood and to discuss some points that, to our opinion, are still open to a development from both the experimental and theoretical point of view. In particular we will limit the discussion to the problem of the radiative recombination of free excitons and the effects that exciton localization at crystal defects has on the recombination mechanisms. Finally a few points concerning the influence of the excitation energy on exciton thermalization and localization will be discussed at the end of our contribution. We will concentrate our attention on photoluminescence (PL) experiments both continuous-wave and time-resolved; details of the experimental apparatuses and sample characteristics have been reported elsewhere $[1,2]$.

Let us start with considering the interaction of an exciton with light. In bulk material it produces mixed modes (polaritons) which are stationary: the exciton cannot radiatively decay into a photon due to the crystal momentum conservation. The picture changes dramatically in QWs, because of the breaking of the translational invariance along the growth axis. An exciton in QWs, with in-plane momentum $\hbar k_{/ /}$less than the resonant 
photon momentum $\hbar q$, interacts with a one-dimensional continuous spectrum of electromagnetic modes resulting in a very efficient radiative decay channels, essentially due to the macroscopic polarization associated with the free exciton wavefunction. The estimated recombination rates for longitudinal and transverse exciton modes are [3] :

$$
\Gamma\left(k_{/ /}\right)=\Gamma_{\mathrm{o}}\left\{\begin{array}{ll}
\frac{\mathrm{q}}{\sqrt{\mathrm{q}^{2}-\mathrm{k}_{/ /}^{2}}} & \mathrm{~T} \text {-mode } \\
\frac{\sqrt{\mathrm{q}^{2}-\mathrm{k}_{/ /}^{2}}}{\mathrm{q}} & \mathrm{L} \text {-mode }
\end{array} \quad(\mathrm{k} / / \leq \mathrm{q})\right.
$$

where $\Gamma_{0}=f * 7.610^{8} \mathrm{sec}^{-1}$ with the oscillator strength $f$ in units (10-5 $\left.\AA-2\right)$. The Z-mode is silent for the ground state heavy-hole $(\mathrm{HH})$ exciton, while is active for the light-hole exciton; the triplet states are always inactive. Typical values of the oscillator strength, for $\mathrm{GaAs} / \mathrm{Al}_{0.3} \mathrm{Ga}_{0.7} \mathrm{As} \mathrm{QWs}$, are $f=30 \div 9010^{-5} \AA^{-2}$ [4]. Therefore the radiative lifetimes at $\mathrm{k}_{/ /}=0$ are of the order of 15-50 $\mathrm{ps}^{(1)}$, depending on the QW parameters (the thinner is the well, the smaller is the lifetime). Note that since $\Gamma\left(k_{/ /}\right)$strongly depends on the exciton in-plane momentum $k_{/ /}$, different lifetimes are then expected inside the angular distribution of the spontaneous emission radiation pattern; furthermore the $\mathrm{T}$-mode decay rate diverges as $k_{\| l} \rightarrow q$. Both these features are washed out when the effect of thermalization, that we will discuss later on, are considered. Finally excitons with kinetic energy higher than $\mathcal{E}=(\hbar \mathrm{q})^{2} / 2 \mathrm{M}(=0.08 \mathrm{meV})$ cannot decay radiatively given the momentum mismatch with respect to the resonant photon. Therefore, in the framework of a pure electromagnetic picture, only a very small fraction of the excitonic states are optically active and their lifetimes are extremely short.

So far we have considered a rigid crystal (no phonons), but a very important role on the exciton radiative kinetics is played by the interaction with the acoustic phonons. This is expected to produce two effects: firstly, instead of a bare exciton one should consider a phonon-dressed exciton; secondly, under the assumption that the scattering rate is larger than the recombination rate, it gives rise to a thermalization of the exciton population. The first effect has not yet been subject of detailed investigations. Qualitatively, scattering by phonons (but also by defects, as we will discuss later on) reduces the exciton coherence length (which should be of the order of the free mean path) making the exciton-photon interaction less effective. Indeed it has been argued that the oscillator strength of the optical active modes (i.e. with kinetic energy $E\left(k_{/ /}\right) \leq \mathcal{E}$ ) should be shared with the exciton states within an energy interval of the order of the homogeneous broadening $\Delta_{h}$ related to the scattering processes $[5,6]$. The radiative rate is consequently reduced by a factor of the order $\mathcal{E} / \Delta_{\mathrm{h}}[5,6]$, as a consequence of the exciton oscillator strength sum rule. A more quantitative estimate can only follow from a microscopic calculation of the excitonphonon interaction; it is worth noting that a similar analysis is still lacking for bulk crystal as well, even if the phonon interaction is fully responsible for the polariton lifetime.

The effects of thermalization on the radiative lifetime have been extensively studied [1-6]. The thermal equilibrium between excitonic states with different in-plane momentum produces a single decay time of the whole exciton population with an average decay rate $\langle\Gamma(T)>$ given by :

$$
<\Gamma(\mathrm{T})\rangle=\frac{\int \mathrm{dE} \rho(\mathrm{E}) \Gamma(\mathrm{E}) \exp \left\{-\mathrm{E} / \mathrm{K}_{\mathrm{B}} \mathrm{T}\right\}}{\int \mathrm{dE} \rho(\mathrm{E}) \exp \left\{-\mathrm{E} / \mathrm{K}_{\mathrm{B}} \mathrm{T}\right\}}
$$

where $\rho(E)$ is the excitonic density of states (a step function for a parabolic and isotropic band). Now, as previously discussed, the radiative modes extend for a very small range of

${ }^{1}$ Intrinsic free exciton lifetimes smaller by a factor two have been predicted in Ref.[5]. 
energy $\delta\left(\delta=\mathcal{E}\right.$ or $\delta=\Delta_{h}$ depending on the picture) and for $K_{B} T>>\delta$ one gets

$$
<\Gamma(\mathrm{T})\rangle=\frac{1}{\mathrm{NK}_{\mathrm{B}} \mathrm{T}} \sum_{\mathrm{i}} \int \Gamma_{\mathrm{i}}(\mathrm{E}) \mathrm{dE}
$$

where $\mathrm{N}$ is the number of exciton states $(\mathrm{N}=4)$ and the index $\mathrm{i}$ runs over the optical active modes ( $i=L, T$ for $H H$ exciton). Due to the sum rule the average rate $\langle\Gamma(T)\rangle$ does not depend on the detailed energy shape of $\Gamma(\mathrm{E})$ and from Eq.(1) one obtains, for $\mathrm{HH}$ excitons:

$$
<\Gamma(\mathrm{T})\rangle=\frac{2 \Gamma_{\mathrm{o}} \mathcal{E}}{3 \mathrm{~K}_{\mathrm{B}} \mathrm{T}}=\frac{f\left(10^{-5} \AA^{-2}\right)}{1793 \mathrm{~T}(\mathrm{~K})} \mathrm{ps}^{-1}
$$

Therefore the thermalization smears out the differences between the lifetime of a bare exciton and of a phonon-dressed exciton [5], the relevant parameter being the integrated radiative rate. Since an increasingly small fraction of the excitons are optically active when raising the temperature, a bottle-neck effect is generated and the lifetime $\tau(T)=1 /\langle\Gamma(T)\rangle$ increases consequently with $\mathrm{T}$. The linear dependence is a peculiarity of the $\mathrm{QW}$ systems; $\mathrm{T}^{3 / 2}$ and $\mathrm{T}^{1 / 2}$ are predicted for mesoscopic structures [7] and quantum wires [8], respectively.

From the experimental point of view the condition $\mathrm{K}_{\mathrm{B}} \mathrm{T} \gg>\delta$ is valid even at cryogenic temperatures $\left(E / K_{\mathrm{B}} \sim 1 \mathrm{~K}\right.$ and $\Delta_{\mathrm{h}} / \mathrm{K}_{\mathrm{B}} \sim 5 \mathrm{~K}$ ). Therefore only non-thermalized or extremely cold excitons can manifest the intrinsic properties of the optical active modes; since other contributions to this Conference are devoted to the experimental study of this subject, here we will only touch a few related points at the end of this paper. On the other hand, Eq. (4) can be directly tested by experiments in a range of easily accessible temperatures.

We report in Fig. 1 the PL decay times of high quality 40,120 and $180 \AA$ single QWs of $\mathrm{GaAs} / \mathrm{Al}_{0.3} \mathrm{Ga}_{0.7} \mathrm{As}$ as a function of temperature; we leave aside the lowest temperatures in order to satisfy the condition $K_{B} T>>\delta$. We refer to the end of this contribution for a brief discussion of the validity of the thermalization hypothesis, while a more detailed analysis can be found in Ref.[2]. A linear fit to the experimental points for all the QWs investigated yields the slopes reported in Table I; the oscillator strength values deduced from Ref.[4] and the slopes predicted according to Eq.(4) are also reported in Table I. We see that the agreement between the theoretical and experimental slopes is indeed good both for the temperature and the well-width dependence. A slight discrepancy is found in the case of the widest well $(180 \AA)$ where the experimental value turns out to be somewhat larger than predicted. In fact for such a well the LH-HH exciton energy difference is so small that the light hole band is already thermally populated at very low temperatures.

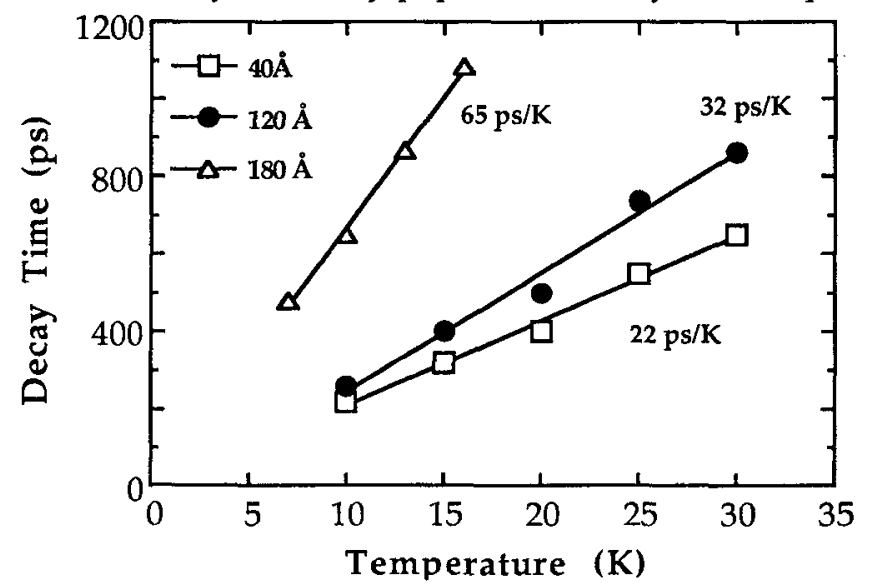

Figure 1: PL decay times as a function of temperature after excitation at the $\mathrm{LH}$ transition energy for single QWs of $\mathrm{GaAs} / \mathrm{Al}_{0.3} \mathrm{Ga}_{0.7} \mathrm{As}$. 
Table I.- Comparison between the experimental and calculated slopes for the temperature dependence of the free exciton lifetimes at low temperatures.

\begin{tabular}{|c|c|c|c|}
\hline $\mathrm{L}_{\mathrm{W}}(\AA)$ & $f\left(10^{-5} \AA^{-2}\right)$ & slope $^{\mathrm{cal}}\left(\mathrm{ps} \mathrm{K}^{-1}\right)$ & slope $^{\text {exp }}\left(\mathrm{ps} \mathrm{K}^{-1}\right)$ \\
\hline \hline 40 & 85 & 21 & 22 \\
\hline 60 & 70 & 26 & 28 \\
\hline 80 & 60 & 30 & 27 \\
\hline 120 & 45 & 40 & 32 \\
\hline 180 & 33 & 54 & 65 \\
\hline
\end{tabular}

Therefore given the smaller oscillator strength of the $\mathrm{LH}$ exciton compared to the $\mathrm{HH}$ exciton, we expect Eq.(4), based on the HH exciton band only, to underestimate the temperature dependence of the exciton lifetime. Other effects not included in Eq.(4), namely exciton ionization, non-negligible populations of the higher subbands and free carrier recombination, strongly influence the recombination kinetics at higher $T$; we refer to Ref.[1] for a discussion of the radiative lifetimes at higher temperatures.

We also remark that PL lifetimes which rise linearly with the temperature have been observed by several other groups [6,9]; however the numerical results for the slopes seem to be dependent on the sample, thus denoting extrinsic effects. A qualitative explanation of this behaviour can be found in presence of interface defects; let us therefore switch to the discussion of the effects of exciton localization.

The interface roughness and compositional fluctuations, practically unavoidable in real QWs, are well-known to provide changes in the QW potential profile, with respect to the simple model of a perfect square well, that can scatter or even localize the excitons. In the last years it has been demonstrated that PL is a very powerful tool for both extracting information on the morphology of the heterointerfaces and studying the dynamics connected with the localization process and the following radiative recombination. In fact, one of the most direct evidence of exciton localization is the red-shift (Stokes shift) of the PL line with respect to the absorption peak, which also provides an estimate of the exciton binding energy at the defect. Similar information can be extracted from the PL linewidth [10]; it has been also claimed recently that the localization reflects itself on the PL exciton lineshape through the presence of an exponential high energy tail in the spectrum [11].

The effect of exciton localization on the radiative recombination time and on its temperature dependence has been recently discussed [5] and we will briefly report the main results. In the framework of a variational method, successfully employed for the analysis of the Stokes shift, it has been shown that lifetimes of the order of $100 \div 300$ ps are expected depending on the well-width and the defect extension and depth. Such values are much larger than the intrinsic lifetime of the free exciton as a consequence of the localization of the exciton wavefunction. In fact there is a similarity with the previously discussed case of the phonon-dressed exciton, although we are now dealing with an extrinsic effect of localization at a given point of the crystal; in the case of the interaction with the phonons, the exciton instead suffers a reduction of the coherence length while being still delocalized over the whole crystal. We also remark that for bound or localized excitons, both in bulk and QWs, the concept of giant oscillator strength is generally used for describing the relatively large radiative rate, compared, for instance, with atomic transitions. In general this is a misleading concept for Wannier excitons [12] and it becomes completely wrong in the QW case where the lifetime of a localized exciton turns out to be one order of magnitude larger than the free exciton lifetime.

We now analyze the effect of thermal equilibrium between localized and free exciton states. The interface defects, with a density per unit of area $N_{D}$, produce a localized state, lying below the free exciton band by the binding energy $\mathrm{E}_{\text {loc }}$, to which corresponds a radiative rate $\Gamma_{l o c}$. Assuming an efficient thermalization between localized and free states, 
that we suppose to be unaffected by the presence of defects, the average radiative rate is given by $\left(K_{B} T>>\mathcal{E}\right)[5]$ :

$$
<\Gamma_{\text {loc }}(\mathrm{T})>=\frac{\mathrm{N}_{\mathrm{loc}} \Gamma_{\mathrm{loc}}+\mathrm{N}_{\mathrm{fr}} \Gamma_{\mathrm{fr}}}{\mathrm{N}_{\mathrm{loc}}+\mathrm{N}_{\mathrm{fr}}}
$$

where $N_{\text {loc }}=N_{D} \exp \left(E_{\text {loc }} / K_{B} T\right), N_{f r}=4 \mathrm{MK}_{B} T / 2 \pi \hbar^{2}$ and $\Gamma_{\text {fr }}$ is the averaged rate for free exciton given by Eq.(4) $\left(\Gamma_{\mathrm{fr}}=2 \Gamma_{\mathrm{o}} \mathcal{E} / 3 \mathrm{~K}_{\mathrm{B}} \mathrm{T}\right)$. At a given temperature $\mathrm{N}_{\mathrm{loc}}$ and $\mathrm{N}_{\mathrm{fr}}$ are proportional to the localized and free exciton populations, respectively.

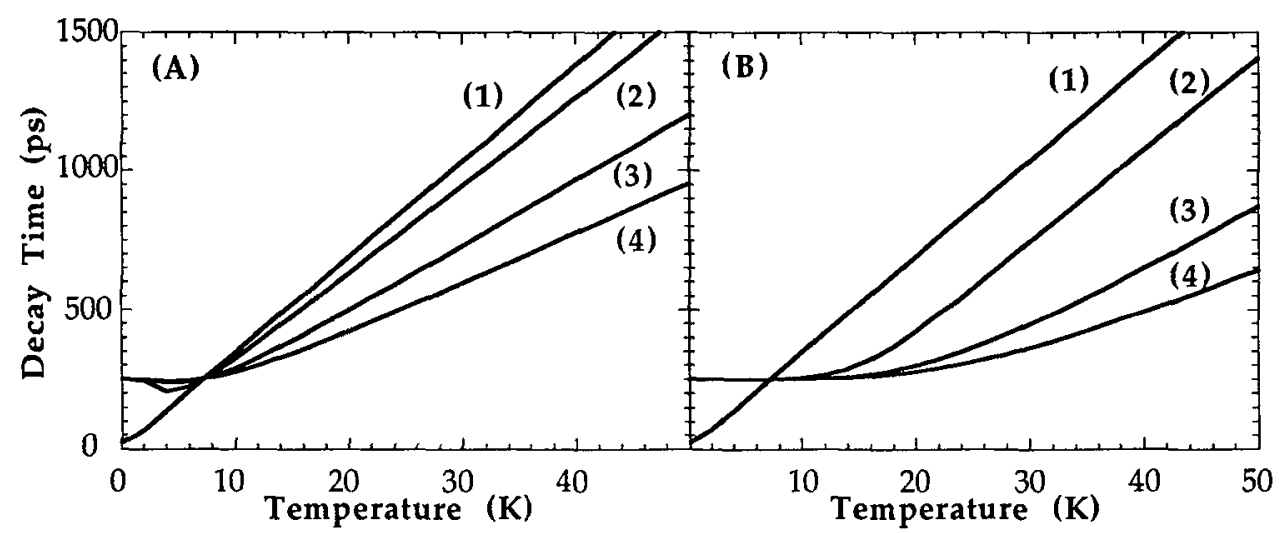

Figure 2: Exciton decay times as a function of temperature from Eq.(5) for the following set of parameters: $1 / \Gamma_{\mathrm{fr}}=25 \mathrm{ps} ; 1 / \Gamma_{\mathrm{loc}}=250 \mathrm{ps}$; curves $(1)-(4)$ refer to $\mathrm{N}_{\mathrm{D}}=0,10^{10}, 510^{10}, 10^{11} \mathrm{~cm}^{-2}$;figures (A) and (B) correspond to $\mathrm{E}_{\mathrm{loc}}=1 \mathrm{meV}$ and $\mathrm{E}_{\mathrm{loc}}=5 \mathrm{meV}$, respectively.

The predictions of Eq.(5) for the dependence of the exciton decay time $\left(1 /<\Gamma_{\text {loc }}(T)>\right)$ on temperature are reported in Fig.(2A) and Fig.(2B) for localization energies $\mathrm{E}_{\mathrm{loc}}=1 \mathrm{meV}$ and $E_{l o c}=5 \mathrm{meV}$, respectively; different lines in the graphs refer to different densities of defects (see the figure caption). Only the temperature interval $\mathrm{T} \leq 50 \mathrm{~K}$ has been considered, due to the relevant role that exciton ionization plays at higher $T$ [1]. As expected the recombination kinetics at low $T$ is dominated by the localized states and the exciton decay time turns out to be $1 / \Gamma_{\text {loc. }}$ More surprising is the fact that the defects, still for small concentration and depth, have an effect on the radiative rate even in the high temperature regime, although the free exciton population largely exceeds the localized exciton population $\left(\mathrm{N}_{\mathrm{fr}}=\mathrm{N}_{\text {loc }}\right.$ essentially at the temperature where the T-dependence of the decay time changes slope). For $K_{B} T>>E_{l o c}$ and $K_{B} T>>\pi \hbar 2 N_{D} / 2 M$ (i.e. $N_{f r}>>N_{D}$ ) Eq.(5) predicts a linear increase of the decay time with raising $T$ (the slope depending on $N_{D}$ ) In fact a meaningful comparison only applies between the population of the optical active modes $(E<\mathcal{E})$ and $\mathrm{N}_{\text {loc }}$, both weighted by the relative radiative rates. The reason for the influence of shallow defects on the high temperature behaviour of the exciton lifetime basically consists in the extremely small energy extension of the optically active states.

For comparison we report in Fig.(3) the experimental PL decay times for several different QWs showing exciton localization with a Stokes shift in the range 2-8 meV. The agreement between the experimental data and the prediction of the model is qualitatively fairly good. The PL lifetimes at $\mathrm{T}=4 \mathrm{~K}$ is of the order of $250 \mathrm{ps}$ and increases sublinearly with increasing temperature. Moreover to the larger QWs, which show a faster increase of the PL decay time, correspond a smaller Stokes shift in agreement with the behaviour reported in Fig.(2A) and Fig.(2B). For a fully quantitative comparison one should know the defect density $\mathrm{N}_{D}$, which strongly modifies the temperature dependence of the excitonic 


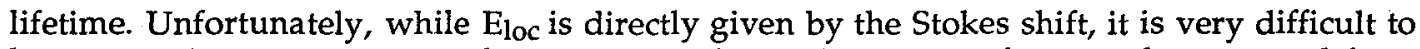
have a precise measurement of $N_{D}$. An experimental estimate of $N_{D}$ can be extracted from the temperature dependence of the Stokes shift which should vanish for $N_{\mathrm{fr}} \Gamma_{\mathrm{fr}} \geq \mathrm{N}_{\mathrm{loc}} \Gamma_{\mathrm{loc}}$. In the case of the QWs reported in Fig.(3) the Stokes shift disappears in the region $40-80 \mathrm{~K}$ : the larger is the well, the lower is the temperature, in agreement with the behaviour reported in Fig.(3).

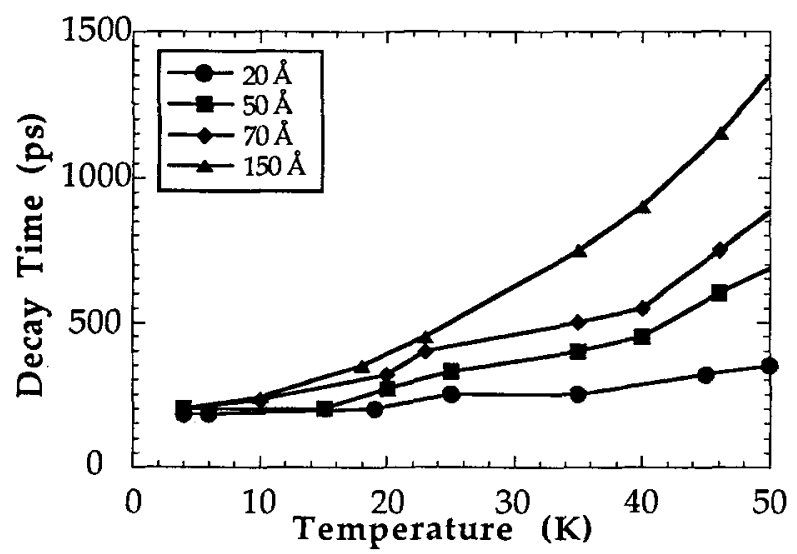

Figure 3: PL decay times as a function of temperature for different $\mathrm{QWs}$ of $\mathrm{GaAs} / \mathrm{Al}_{0.3} \mathrm{Ga}_{0.7} \mathrm{As}$. The lines are only guides for the eye.

To conclude this part we would like to remark a weak aspect of the model, reflected in Eq.(5), that is, the assumption that the free states are unaffected by the presence of the defects. In fact free excitons will be scattered by the interface roughness with a consequent reduction of the coherence length $[5,13]$; one can therefore apply to this problem the same qualitative considerations we have done for the phonon-exciton interaction. We believe that these are two very important, but still open, problems in the exciton recombination kinetics.

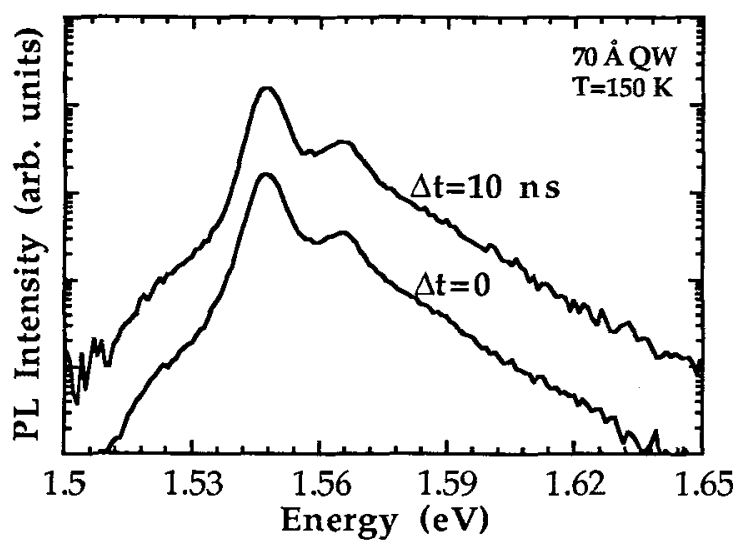

Figure 4: Time resolved PL spectra of a $70 \AA \mathrm{GaAs} / \mathrm{Al}_{0.3} \mathrm{Ga}_{0.7} \mathrm{As} \mathrm{QW}$, at $\mathrm{T}=150 \mathrm{~K}$ for two different delay times $\Delta t$ from the pulse excitation; the absence of any dynamics in the whole PL band denotes thermalization.

Let us now, at the end of this contribution, briefly discuss the validity of Eqs.(4)-(5) as far as the main underlying hypothesis, namely the thermalization, is concerned. Two different aspects can be stressed: on one hand, the experimental evidences and conditions 
for the applicability of such hypothesis when interpreting the experimental data; on the other hand, the ways for avoiding thermalization in order to measure the intrinsic properties of the exciton optical active modes.

As already remarked, thermal equilibrium between different states implies a single decay time of the whole PL band(2); therefore a very direct evidence for thermalization can be found by the analysis of PL time resolved spectra, which should not show any temporal dynamics. A clear example is reported in Fig.(4), where several different contributions in thermal equilibrium at $\mathrm{T}=150 \mathrm{~K}$ can be resolved in the PL spectra, namely heavy/light excitons and free carrier recombination [1]; nevertheless we measure the same lifetime over a PL band spanning more than $100 \mathrm{meV}$.

At low temperature and/or near resonant excitation, instead, the thermalization hypothesis is not always verified. Indeed the low temperature dynamics inside the time resolved PL spectra has been extensively used for identifying different contributions to the recombination. A dramatic effect, for example, is found on the exciton recombination kinetics for resonant excitation at the $\mathrm{HH}$ exciton energy, as reported in Fig. $(5 \mathrm{~A})$ where the PL decay is shown for both $E_{\text {exc }}=E_{H H}$ and $E_{e x c}=E_{L H}$. The different behaviour cannot be associated to extrinsic Rayleigh scattering due to the much higher PL measured intensity compared to the laser light and given the completely different time profile of the two signals. It should also be noted that, in agreement with the PLE spectra, not reported here; the PL integrated intensity is basically the same when exciting resonant with the $\mathrm{HH}$ - or LH-exciton transition: it is only the recombination kinetics which is completely different. The reason of this effect seems to be the photogeneration of very cold unthermalized excitons that therefore decay with a shorter lifetime [16]. We also refer to other contributions to this Conference for a more exhaustive analysis of this point [17].
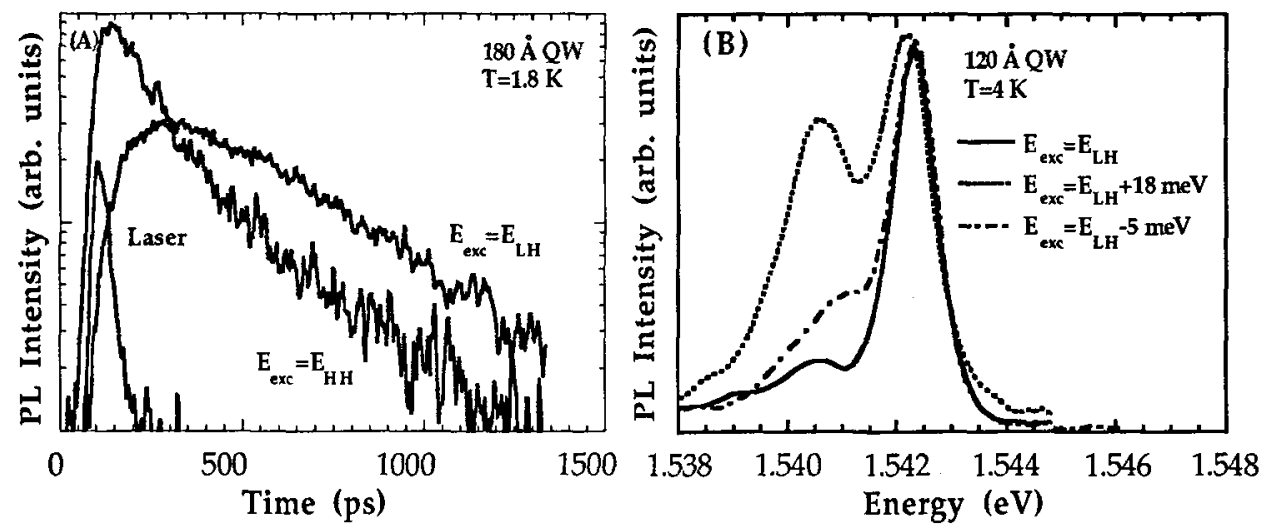

Figure (5) Examples, in $\mathrm{GaAs} / \mathrm{Al}_{0.3} \mathrm{Ga}_{0.7} \mathrm{As} \mathrm{QWs}$, of different aspects connected with thermalization at low $T$ under different excitation energies $E_{\text {exc. }}$. $(A) P L$ decay for different excitation energies; as a comparison the laser scattered light, for LH excitation, is also reported. (B) Continuous wave PL spectra for different excitation energies: resonant excitation on the LH exciton reduces the localization effects.

As a further example of the dependence of the recombination kinetics on the excitation energy, we show in Fig. (5B) how resonant excitation at the LH transition energy produces a strong enhancement in the generation of the free HH exciton (dominant PL peak) compared to the localized excitons (low-energy PL band). As a consequence, resonant excitation on the LH excitons has been successfully used for both reducing the effect of

2 Note that there are some exception to this rule: for instance, at thermal equilibrium, the biexciton band decays faster than the exciton line by a factor two[14]. 
localization on the study of recombination kinetics [2] and for enhancing the biexciton formation[15].

In summary, we have tried to give an overview, without any ambition of completeness, of some relevant topics concerning the exciton recombination dynamics we have studied in these last few years. In particular the problem of the exciton intrinsic radiative lifetime has been discussed in detail together with the effects that localization at defects has on the recombination kinetics. A good agreement is eventually found between the theoretical predictions and the experimental data. Finally we have pointed out some problems we believe to be not completely solved, namely the effects of phonons and/or interface defect scattering on the exciton lifetimes.

Acknowledgments: We warmly thank Drs. Anna Vinattieri, Luca Carraresi and Philippe Roussignol for participating to large parts of the work reported here.

\section{References:}

[1] M. Gurioli, A. Vinattieri, M. Colocci, C. Deparis, J. Massies, G. Neu, A. Bosacchi, and S. Franchi, Phys. Rev. B 44, (1991) 3115-3124;

M. Colocci, M. Gurioli, and A. Vinattieri, Physica Scripta T39 ( (1991) 211-216.

[2] J. Martinez-Pastor, A. Vinattieri, L. Carraresi, M. Colocci, Ph. Roussignol, and G. Weimann, Phys. Rev.B 47, (1993) 10456-10460.

[3] L.C. Andreani, F. Tassone, and F. Bassani, Solid State Commun. 77, (1991) 641-648.

[4] L.C. Andreani, and A. Pasquarello, Phys. Rev. B 42, (1990) 8928-8935.

[5] D. S. Citrin, Phys. Rev. B 47, (1993) 3832-3841

[6] J. Feldmann, G. Peter, E.O. Göbel, P. Dawson, K. Moore, G. Foxon, and R.J. Elliot, Phys. Rev. Lett. 59, 2337 (1987); 60, (1988) 243-246.

[7] L.C. Andreani, A. d'Andrea, and R. del Sole, Phys. Lett. A 168, (1992) 451-459.

[8] H. Akiyama, S. Koshiba, T. Someya, K. Wada, H. Noge, Y. Nakamura, T. Inoshita, A. Ahimizu, and H. Sakaki, to be published.

[9] H. Hillmer, A. Forchel, S. Hansmann, M. Morohashi, E. Lopez, H. P. Meier and K. Ploog, Phys. Rev. B 29, (1989) 10901-10910; H. Matsueda and K. Hara, Appl. Phys. Lett. 55, (1989) 362-364.

[10] F. Yang, M. Wilkinson, E. J. Austin, and K. P. O'Donnell, Phys. Rev. Lett. 70, (1993) 323-326.

[11] R. F. Schnabel, R. Zimmermann, D. Bimberg, H. Nickel, R. Losch, and W. Schlapp, Phys. Rev. B 46, (1992) 9873-9876

[12] L. C. Andreani, to be published.

[13] V. Srinivas, J. Hryniewicz, Y.J. Chen, and E.C. Wood, Phys. Rev. B 46, (1992) 1019310196.

[14] S. Charbonneau, T. Steiner, M. L. W. Thewalt, E. S. Koteles, J. Y. Chi, and B. Elman, Phys. Rev. B 38, 3583-3586.

[15] D. J. Lovering, R. T. Phillips, G. J. Denton, G. W. Smith, Phys. Rev. Lett. 68, (1992) 18801883

[16] B. Deveaud, F. Clérot, N. Roy, K. Satzke, B. Sermage, and D.S. Katzer, Phys. Rev. Lett. 67, (1991) 2355-2358.

[17] B. Sermage, S. Long, B. Deveaud, and D. S. Katzer; "Exciton recombination dynamics in quantum wells", to be published in Proc. of Third Int. Conf. on "Optics of Excitons in Confined System" Montpellier August 30-September 2 1993;

A. Vinattieri, J. Shah, T. C. Damen, D. S. Kim, L. P. Pfeiffer, and L. J. Sham, Picosecond dynamics of resonantly excited excitons in GaAs quantum wells", ibidem. 